\title{
The 46th Annual Meeting of the European Society for Blood and Marrow Transplantation: Organising Committee
}

C Springer Nature Limited 2020

29 August - 1 September, 2020 Virtual Meeting

Modified and published with permission from https://www.ebmt.org/annual-meeting

Sponsorship Statement: Publication of this supplement is sponsored by the European Society for Blood and Marrow Transplantation.

\section{Local Organizing Committee}

Rafael F. Duarte, Congress President

Anna Sureda, Scientific Chair

Isabel Salcedo, Local Nurses Co-Chair

Julia Ruiz, Local Nurses Co-Chair

Jorge Sierra

Ramón García-Sanz

Carlos Solano

Marta González-Vicent

Rafael de la Cámara

Cristina Díaz de Heredia

Sebastián Galeano

Gregorio Jaimovich

Alejandro Madrigal

Joaquín Martínez

José $\mathbf{M}^{\mathrm{a}}$ Moraleda

Antonio Pérez-Martínez

Sergio Querol

Álvaro Urbano-Ispizua

\section{Congress Advisory Group}

Miguel Ángel Sanz

Christian Chabannon

Manuel Abecasis

Peter Bader

Pavel Jindra

Petr Sedlacek

Mahmoud Aljurf

Arnon Nagler
Alejandro Madrigal

Hermann Einsele

\section{EBMT Board of Association}

President: Nicolaus Kröger, Germany

Secretary: Rafael Duarte, Spain

Treasurer: Harry Dolstra, The Netherlands

Executive Director: Andreu Gusi, Spain

Scientific Council Chair: Mohamad Mohty, France

Scientific Council Vice-Chair: Christian Chabannon, France

Scientific Council Representative with Porfolio Registry:

John Snowden, UK

Scientific Council Representative with Portfolio Education:

Grzegorz W. Basak, Poland

President of the EBMT Nurses Group: John Murray, UK

Presidents of the 2020 EBMT Annual Meeting: Rafael

Duarte, Spain

\section{Scientific Council}

Acute Leukemia Working Party Chair: Mohamad Mohty, France

Severe Aplastic Anaemia Working Party Chair: Régis Peffault de Latour, France

Autoimmune Diseases Working Party Chair: John Snowden, UK

Chronic Malignancies Working Party Chair: Ibrahim Yakoub-Agha, France 
Cellular Therapy \& Immunobiology Working Party Chair: Christian Chabannon, France

Inborn Errors Working Party Chair: Arjan Lankester, The Netherlands

Infectious Diseases Working Party Chair: Jan Styczynski, Poland

Transplant Complications Working Party Chair: Grzegorz

Basak, Poland

Lymphoma Working Party Chair: Silvia Montoto, UK

Paediatric Diseases Working Party Chair: Selim Corbacioglu, Germany

\section{EBMT Nurses Group Board \& Scientific Committee}

Nurses Group President: John Murray, UK

Nurses Group President Elect: Michelle Kenyon, UK

Nurses Group Secretary: Daphna Hutt, Israel

Local Nurse Representative of the 2020 EBMT Annual Meeting: Julia Ruiz, Spain

Local Nurse Representative of the 2020 EBMT Annual Meeting: Isabel Salcedo, Spain

\section{Data Management Group}

Debra Gordon, UK

Shelley Hewerdine, UK
Khrystyna Valkiv, UK

Silvia Zaccagnino, UK

\section{Statistics Group}

Chair: Simona Iacobelli, Italy

Myriam Labopin, France

Richard Szydlo, UK

Liesbeth de Wreede, The Netherlands

Ariane Boumendil, France

\section{Quality Management Group}

Chair: Renza Monteleone, Italy

Dieter Klarmann, Germany

Ilknur Kozanoglu, Turkey

Mara Magri, Italy

Nick van Sinderen, The Netherlands

Anne Emmet, UK

Julie Dolva, Norway

Songul Durmaz, Turkey

Simon Hack, UK

Phuong Huynh, Brussels

Olga López Villar, Spain 